OPEN ACCESS

Edited by:

Chris Keyworth,

The University of Manchester

United Kingdom

Reviewed by:

Michela Rimondini,

University of Verona, Italy

Sara Marelli,

San Raffaele Hospital (IRCCS), Italy

*Correspondence:

Giulia Marton

giulia.marton@ieo.it

${ }^{\dagger}$ These authors share first authorship

Specialty section:

This article was submitted to Psychology for Clinical Settings,

a section of the journal

Frontiers in Psychology

Received: 29 July 2020

Accepted: 22 September 2020

Published: 15 October 2020

Citation:

Marton G, Vergani L, Mazzocco K,

Garassino MC and Pravettoni $G$ (2020) 2020s Heroes Are Not

Fearless: The Impact of the COVID-19

Pandemic on Wellbeing and Emotions of Italian Health Care Workers During

Italy Phase 1.

Front. Psychol. 11:588762.

doi: 10.3389/fpsyg.2020.588762

\section{0s Heroes Are Not Fearless: The Impact of the COVID-19 Pandemic on Wellbeing and Emotions of Italian Health Care Workers During Italy Phase 1}

\author{
Giulia Marton ${ }^{1,2 * \dagger}$, Laura Vergani ${ }^{1,2 \dagger}$, Ketti Mazzocco ${ }^{1,2}$, Marina Chiara Garassino ${ }^{3}$ and \\ Gabriella Pravettoni ${ }^{1,2}$ \\ ${ }^{1}$ Applied Research Division for Cognitive and Psychological Science, IEO, European Institute of Oncology IRCCS, Milan, \\ Italy, ${ }^{2}$ Department of Oncology and Hemato-Oncology, University of Milan, Milan, Italy, ${ }^{3}$ Thoracic Oncology Unit, Division of \\ Medical Oncology, Foundation IRCCS-Italian National Cancer Institute, Milan, Italy
}

Objective: The study aimed to investigate the mental health and emotional reaction of physicians working during phase 1 of the COVID-19 pandemic in Italy.

Methods: A total of 458 Italian Health Care Workers (HCWs) working during phases 1 of the COVID-19 outbreak were voluntarily enlisted in the study and recruited with the snowball technique through an online survey. We examined our variables with the General Health Questionnaire - 12 and with Visual Analog Scales.

Results: The sample has a high level of psychological distress 21.26 (SD = 4.46), the emotional reaction was characterized by high level of fear for family members and cohabitants $(M=77.67, S D=27.16)$ and patients $(M=67.16, S D=27.71)$. Perceived control, fear for patients, and for family members and cohabitants, feeling alone and anger all contribute to a decreased mental health in Italian physicians $\left(R^{2}=0.285, \mathrm{p}<0.001\right)$

Conclusion: Italian HCWs' mental health and emotional reaction have to be considered to prevent high risk of burnout and post-traumatic stress disorder (PTSD). It becomes pivotal in the next months to implement a tailored psychological intervention to take care of HCWs and to prevent costly consequences for them, patients, and the healthcare system.

Keywords: health care workers, COVID-19, emotional reaction, health care workers wellbeing, distress

\section{INTRODUCTION}

On the 11th of March 2020, the World Health Organization characterized COVID-19 as a pandemic. In Italy the situation was already very serious and, as one of the first States to be affected by this outbreak, the spread of the disease was at its peak, with the national lockdown imposed on the 9th of March. Only on the 18th of May phase 1 ended, with the restrictive measure of lockdown loosen and the healthcare system registering a break from the emergency. COVID-19 spreads rapidly and can cause severe symptoms, giving a lot of pressure to the National Health 
System (Shanafelt et al., 2020). Health care workers' (HCW) workload was very demanding, affected also by suspension of days of leave and rest. Moreover, the strict contact with infected patients and the risk of being infected themselves increase the physical and psychological difficulties that HCWs have to face: the fear is not limited to the possibility to get the virus, but also to the possibility to take it home and infect their families and other people as proven during other pandemics in the past (Maunder et al., 2006). At the beginning of July 2020, more than 1800 HCWs have died because of the COVID-19 (MEDSCAPE, 2020). Also, the limited number of beds in the intensive care units and the dramatically increased number of patients needing intubation, imposed HCWs the responsibility to choose which patients to cure (Rosenbaum, 2020). Such a dilemma accumulated with the aforementioned factors of psychological distress. Studies conducted in past comparable situations showed that the stressful situation, the workload, and the high responsibility affect the psychological wellbeing of HCWs, with acute and chronic consequences (Bai et al., 2004; Chen et al., 2005; Maunder et al., 2006; Khalid et al., 2016; Lai et al., 2020; Li et al., 2020; Zhu et al., 2020). Post traumatic stress, insomnia, depressive, and anxiety symptoms are often reported by HCWs during pandemic and epidemic situations (Preti et al., 2020). During the SARS epidemic, quarantine was associated with emotional distress in HCWs as well as feelings of fear to contract the disease, worry for the family and isolation, the stress in the workspace and stigma of possibly being contagious (Bai et al., 2004; Maunder et al., 2006). Under these conditions, working in a hospital that treated SARS patients led to high burnout levels, psychological distress and post-traumatic stress disorder (PTSD) (Chen et al., 2005). During the MERS-CoV outbreak in 2014, HCWs experienced fear for personal safety and fear for their colleagues and families (Khalid et al., 2016). In China, the first country to be affected by COVID19, HCWs working with COVID-19 patients reported various symptoms of psychological distress, like anxiety, insomnia and depression (Huang and Zhao, 2020; Lai et al., 2020; Zhu et al., 2020). A preliminary study on trauma during the COVID-19 pandemic (Li et al., 2020) suggested that HCW may experience vicarious traumatization due to the frequent experience of seeing patients dying without having their lovedones near them.

But what are the main factors that affect the psychological distress of HCWs during COVID-19 pandemic? The aim of this study was to explore the mental health and emotional reactions of Italian HCW involved in phase 1 of the COVID-19 pandemic. Based on previous studies conducted during similar events, we hypothesized a decreased mental health and emotional distress affecting Italian HCW during phase 1 of the COVID19 pandemic.

\section{METHODS}

For this observational study, we recruited a sample of 458 HCW working during the first phases of the COVID-19 Italian outbreak, through HCWs mailing lists, social media and snowball recruitment. For the considered population, a minimum sample of 400 respondents allows having a certainty measure with a confidence level of $95 \%$ and a confidence interval of $5 \%$ (Hill, 1998). Recruitment started on the 24th of March until the 13th of May and focused on phase 1 of the COVID-19 Italian emergency.

The sample comprised HCWs working all over Italy during the Pandemic, with a mean age of 43.46 years $(S D=10.22$; range: $25-70$ years), a mean of $15.03(\mathrm{SD}=10.23$ ) years of working experience, and a mean of 36.30 ( $\mathrm{SD}=17.73$ ) hours of work per week, with an average of 1.63 days of rest per week $(\mathrm{SD}=0.84)$.

Our sample was composed by $79 \%$ (362 out of 458 ) physicians, $9.8 \%$ (45 out of 458 ) nurses, $5.2 \%$ (24 out of 458 ) technicians, $1.7 \%$ ( 8 out of 458 ) psychologists, $0.7 \%$ ( 3 out of 458 ) OSS, $0.7 \%$ (3 out of 458 ) OTA, $0.7 \%$ (3 out of 458 ) volunteers, $0.4 \%$ (2 out of 458 ) pharmacists, $0.2 \%$ ( 1 out of 458 ) obstetricians and $1.5 \%$ (7 out of 458) other kind of HCWs.

The study was approved by the European Institute of Oncology ethics committee (R1185/20-IEO 1248). Participants provided written informed consent before being asked to fill in an online survey characterized by a standardized questionnaire to measure the mental health status (the 12-item General Health Questionnaire, GHQ; Goldberg et al., 1997), and Visual Analog Scales (VAS) to assess personal experience associated with the situation. In particular, general distress, fear for themselves, their family members and cohabitants and their patients, the anger felt in this period, the perceived level of loneliness and the perceived level of abandonment by the Institutions were assessed. Given the association between perception of control on the situation and the presence of distress (Bhanji et al., 2016), perceived control on the situation was also measured with a VAS. The 12-item GHQ was characterized by 4-point Likert scale answers, with low scores indicating a good mental health status and high scores indicating a bad mental health status. Scores above the threshold of 13/14 indicate the presence of psychological distress (Piccinelli et al., 1993; Goldberg et al., 1997). The VAS were on a range from 0 (not at all) to 100 (completely), with higher values indicating a worse condition except perceived control of the situation. Finally, questions were asked on workload-related information (average number of rest days per week in this period and average working hours per week in this period) and socio-demographic information (years of working experience and age).

We performed descriptive statistics (mean and standard deviation) and, considering a statistical significance of $p>0.05$, we performed a bivariate correlational and a stepwise backward regression analysis on collected data. We performed our analysis with SPSS 26.

\section{RESULTS}

Descriptive statistics for GHQ-12 scores and Visual Analog Scales for all 458 participants are reported in Table 1. The mean score for GHQ-12 was $21.26(\mathrm{SD}=4.46)$, indicating a 
generally high level of psychological distress. The high score in psychological dysfunction was confirmed by physicians' perceived distress directly measured with VAS $(M=67.90$, $\mathrm{SD}=23.16, r=0.54, p<0.01$ ).

Regarding specific emotional reactions due to the COVID19 emergency, participants indicated high levels of fear for family members and cohabitants $(M=77.67, \mathrm{SD}=27.16)$ and fear for patients $(M=67.16, \mathrm{SD}=27.71)$. Instead, fear for themselves received a significantly lower score $(M=48.59$, $\mathrm{SD}=30.98)$ compared to the experience of fear for others fears for family members and cohabitants and for patients [respectively $t(457)=-20.55, p<0.001$ and $t(457)=-11.08$, $p<0.001)$; moreover, fear for family members was significantly higher than fear for patients $[t(457)=8.14, p<0.001]$. Perceived control related to the situation was the lowest score $(M=41.21$, $\mathrm{SD}=26.85)$. Correlation analysis demonstrated a significant negative association between perceived control of the situation and emotional reactions $(p<0.01)$. A negative correlation was found also between perceived control and general mental health $(p<0.01)$ and general distress $(p<0.01)$.

A stepwise backward regression analysis was performed to analyze the predictive effect of fear (for themselves, for family members and cohabitants and for patients), anger, feeling alone, perception to feel abandoned and perceived control on mental health. The final model included perceived control, fear for patients and family, feeling alone and anger as significant predicting factors, explaining $28.5 \%$ of the variability in the total GHQ-12 score $\left(R^{2}=0.285, p<0.001\right)$. Of these variables, feeling alone significantly made the largest contribution in the GHQ12 score $(\beta=0.221)$, followed by anger and fear for family members and cohabitants, fear for patients, and perceived control of the situation (respectively: $\beta=0.176 ; \beta=0.159 ; \beta=0.153$; $\beta=-0.119$ ).

Regression analysis showed also a predictive role of years of experience, feeling alone, fear for patients, and fear for themselves on perceived control $\left(R^{2}=0.062, p<0.001\right)$, with a lower perceived control in presence of higher levels of feeling alone $(\beta=-0.117)$, fear for patients $(\beta=-0.081)$ and fear for themselves $(\beta=-0.099)$. On the contrary, years of working experience significantly affected the perceived control $(\beta=0.133$, $p<0.05$ ), with more years being associated with a higher perceived control.

\section{DISCUSSION}

During COVID-19 pandemic's phase 1, HCWs' life was surrounded by fear. Usually, HCWs are afraid of being blamed and punished but in this period a new fear is added (Gorini et al., 2012). They fear for their patients, and when they finish the endless time in the hospital, they go back to their home and they are afraid to infect families or cohabitants. The fear affects directly the mental health status, and also the perceived control on the situation that, in turn, influences the HCWs' psychological state. Despite fear being a predictive factor of mental health and distress, feeling alone and anger emerged as relevant emotions affecting HCWs' psychological wellbeing. Our results are in line

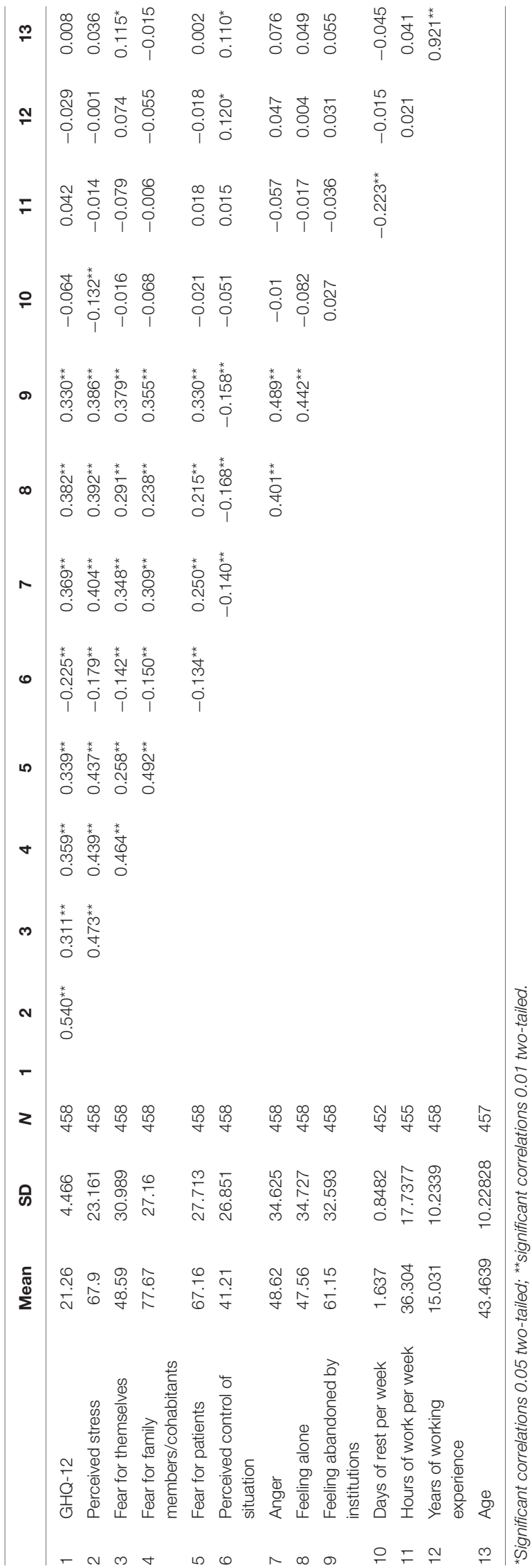


with previous studies about these themes (Sadler and Weiss, 1975; Simard et al., 2013). The relevance of loneliness as a contributor to mental health is confirmed by previous studies showing its predictive role in the development and maintenance of depressive and anxiety symptoms (Wang et al., 2018; Hill and Hamm, 2019). Moreover, loneliness has been found to have impact on other chronic and various diseases: for example, cognitive decline (Shankar et al., 2013), cardiovascular diseases (Herlitz et al., 1998; Sorkin et al., 2002; Hawkley et al., 2010), cancer (Antoni et al., 2006), and inflammatory diseases (Luanaigh and Lawlor, 2008).

Our study links primary emotions with a cognitive aspect: the perception of lack of control. Considering the stress and negative emotions, together with the perceived difficulties in controlling the situation, it is not surprising that these findings are related to mental health.

In COVID-19's phase 1, the pick of infected people was at its maximum and HCWs had to make rapid and often ethically challenging decisions on who and how to care (Wallace et al., 2020). Usually, professionals' decisionmaking priorities should consider patient preferences (Marton et al., 2020; Monzani et al., 2020) to empower patients to reach the preferred decision (Arnaboldi et al., 2020). In this period, however, the low availability of ventilators compared to the high number of critical patients required HCWs to make life-or-death decisions (Rosenbaum, 2020). This might cause "decision fatigue," a psychologically taxing phenomenon originating from the evaluation of pros and cons to make a good decision in the context of high potential risks (Baumeister et al., 1998) that, if not managed, will lead to higher distress (Chen et al., 2018). Moreover, to adapt to the complex environment, HCWs use heuristics that become inevitable (Mazzocco and Cherubini, 2010), and lead to mistakes. On top of that, preliminary studies on trauma during the COVID-19 pandemic (Li et al., 2020) suggested that HCWs may experience vicarious traumatization and emotional dissociation from what they are seeing and experiencing (Masiero et al., 2020): this may lead to PTSD if not timely managed. PTSD may have tremendous consequences and should be monitored not only in the patients' population (Arnaboldi et al., 2014) but also in the HCWs' one.

Perceived lack of control, high level of stress, negative emotions - all symptoms retrieved in our sample - are risk factors for Burnout Syndrome. Potential consequences of not managed distressed conditions will have implications not only for HCWs (Suñer-Soler et al., 2014) but also for patients and health systems: physical problems, diminished job satisfaction, less quality of care, absenteeism, negative attitude. It is fundamental, then, to implement actions and interventions to take care of physicians and HCWs during and after the emergency, to prevent a more costly situation.

The study presents some limitations. The sample only comprehends Italian HCWs, making the results not comprehensive of possible different findings of other nationalities. However, we think that the Italian experience remains an interesting context to consider. Another limit is that our model only explains $28.5 \%$ of HCW's mental health. It is advisable that future research should consider other factors that could explain psychological distress in a more comprehensive way (i.e., individual aspects, the personality of the HCWs).

We also did not consider the working environment of the HCWs that could have caused a difference in how the COVID-19 outbreak has impacted the professionals. Some areas of Italy were more impacted than others and some hospital wards became COVID-19 specific. HCWs that worked in such highly impacted hospitals could have been more affected than others. In particular, we assume the different settings to have an impact on the decision fatigue experienced by the HCWs and on their emotional reactions that we mentioned above.

Among other results, we retrieved a detrimental emotional reaction of HCWs; moreover, perceived control, fear for patients and for families, feeling alone and anger, predict mental health. To mitigate these symptoms and to prevent their evolution in chronic diseases, it is pivotal to implement tailored psychological interventions that help HCWs to develop and improve skills in order to manage their emotional reactions, cope to the stressful working environment and foster their psychological well-being (Masiero et al., 2018).

\section{DATA AVAILABILITY STATEMENT}

The datasets presented in this article are available from the corresponding author on reasonable request. Requests to access the datasets should be directed to giulia.marton@ieo.it.

\section{ETHICS STATEMENT}

The studies involving human participants were reviewed and approved by the European Institute of Oncology ethics committee (R1185/20-IEO 1248). The patients/participants provided their written informed consent to participate in this study.

\section{AUTHOR CONTRIBUTIONS}

GM, LV, and KM planned and conducted the study and drafted the manuscript. GP and MG supervised all the processes, provided critical guidance, and revised the manuscript. All authors contributed to the article and approved the submitted version.

\section{FUNDING}

This work was partially supported by the Italian Ministry of Health with Ricerca Corrente and $5 \times 1000$ funds.

\section{ACKNOWLEDGMENTS}

GM and LV are Ph.D. students within the European School of Molecular Medicine (SEMM). 


\section{REFERENCES}

Antoni, M. H., Lutgendorf, S. K., Cole, S. W., Dhabhar, F. S., Sephton, S. E., McDonald, P. G., et al. (2006). The influence of bio-behavioural factors on tumour biology: pathways and mechanisms. Nat. Rev. Cancer 6, 240-248. doi: $10.1038 / \mathrm{nrcl} 820$

Arnaboldi, P., Lucchiari, C., Santoro, L., Sangalli, C., Luini, A., and Pravettoni, G. (2014). PTSD symptoms as a consequence of breast cancer diagnosis: clinical implications. SpringerPlus 3:392. doi: 10.1186/2193-1801-3-392

Arnaboldi, P., Oliveri, S., Vergani, L., Marton, G., Guiddi, P., Busacchio, D., et al. (2020). The clinical-care focused psychological interview (CLiC): a structured tool for the assessment of cancer patients' needs. Ecancermedicalscience 14:1000. doi: 10.3332/ecancer.2020.1000

Bai, Y., Lin, C. C., Lin, C. Y., Chen, J. Y., Chue, C. M., and Chou, P. (2004). Survey of stress reactions among health care workers involved with the SARS outbreak. Psychiatric Serv. 55, 1055-1057. doi: 10.1176/appi.ps.55.9.1055

Baumeister, R. E., Bratslavsky, E., Muraven, M., and Tice, D. M. (1998). Ego depletion: is the active self a limited resource? J. Pers. Soc. Psychol. 74, 1252-1265.

Bhanji, J. P., Kim, E. S., and Delgado, M. R. (2016). Perceived control alters the effect of acute stress on persistence. J. Exp. Psychol. General 145, 356-365. doi: $10.1037 / \mathrm{xge} 0000137$

Chen, C. S., Wu, H. Y., Yang, P., and Yen, C. F. (2005). Psychological distress of nurses in Taiwan who worked during the outbreak of SARS. Psychiatric Serv. 56, 76-79. doi: 10.1176/appi.ps.56.1.76

Chen, C. Y., Rossignac-Milon, M., and Higgins, E. T. (2018). Feeling distressed from making decisions: assessors' need to be right. J. Pers. Soc. Psychol. 115, 743-761. doi: 10.1037/pspp0000181

Goldberg, D. P., Gater, R., Sartorius, N., Ustun, T. B., Piccinelli, M., Gureje, O., et al. (1997). The validity of two versions of the GHQ in the WHO study of mental illness in general health care. Psychol. Med. 27, 191-197. doi: 10.1017/ S0033291796004242

Gorini, A., Miglioretti, M., and Pravettoni, G. (2012). A new perspective on blame culture: an experimental study. J. Eval. Clin. Pract. 18, 671-675. doi: 10.1111/j. 1365-2753.2012.01831.x

Hawkley, L. C., Thisted, R. A., Masi, C. M., and Cacioppo, J. T. (2010). Loneliness predicts increased blood pressure: 5-Year cross-lagged analyses in middle-aged and older adults. Psychol. Aging 25, 132-142. doi: 10.1037/a0017805

Herlitz, J., Wiklund, I., Caidahl, K., Hartford, M., Haglid, M., Karlsson, B. W., et al. (1998). The feeling of loneliness prior to coronary artery bypass grafting might be a predictor of short-and long-term postoperative mortality. Eur. J. Vascul. Endovascul. Surg. 16, 120-125. doi: 10.1016/s1078-5884(98)80152-4

Hill, E. M., and Hamm, A. (2019). Intolerance of uncertainty, social support, and loneliness in relation to anxiety and depressive symptoms among women diagnosed with ovarian cancer. Psycho-Oncology 28, 553-560. doi: 10.1002/pon. 4975

Hill, R. (1998). What sample size is "enough" in internet survey research. Int. Comput. Technol.: An Electronic J. 21st Century 6, 3-4.

Huang, Y., and Zhao, N. (2020). Generalized anxiety disorder, depressive symptoms and sleep quality during COVID-19 outbreak in China: a web-based cross-sectional survey. Psychiatry Res. 288:112954.

Khalid, I., Khalid, T. J., Qabajah, M. R., Barnard, A. G., and Qushmaq, I. A. (2016). Healthcare workers emotions, perceived stressors and coping strategies during a MERS-CoV outbreak. Clin. Med. Res. 14, 7-14. doi: 10.3121/cmr.2016.1303

Lai, J., Ma, S., Wang, Y., Cai, Z., Hu, J., Wei, N., et al. (2020). Factors associated with mental health outcomes among health care workers exposed to coronavirus disease 2019. JAMA Network Open 3:e203976. doi: 10.1001/jamanetworkopen. 2020.3976

Li, Z., Ge, J., Yang, M., Feng, J., Qiao, M., Jiang, R., et al. (2020). Vicarious traumatization in the general public, members, and non-members of medical teams aiding in COVID-19 control. Brain Behav. Immun. 88, 916-919. doi: 10.1016/j.bbi.2020.03.007

Luanaigh, C. Ó, and Lawlor, B. A. (2008). Loneliness and the health of older people. Int. J. Geriatric Psychiatry 23, 1213-1221. doi: 10.1002/gps.2054

Marton, G., Pizzoli, S. F. M., Vergani, L., Mazzocco, K., Monzani, D., Bailo, L., et al. (2020). Patients' health locus of control and preferences about the role that they want to play in the medical decision-making process. Psychol. Health Med. 23, 1-7. doi: 10.1080/13548506.2020.1748211

Masiero, M., Cutica, I., Russo, S., Mazzocco, K., and Pravettoni, G. (2018). Psycho-cognitive predictors of burnout in healthcare professionals working in emergency departments. J. Clin. Nurs. 27, 2691-2698. doi: 10.1111/jocn.14376

Masiero, M., Mazzocco, K., Harnois, C., Cropley, M., and Pravettoni, G. (2020). From individual to social trauma: sources of everyday trauma in Italy, the US and UK during the Covid-19 pandemic. J. Trauma Dissoc. 1-7. doi: 10.1080/ 15299732.2020.1787296

Maunder, R. G., Lancee, W. J., Balderson, K. E., Bennett, J. P., Borgundvaag, B., Evans, S., et al. (2006). Long-term psychological and occupational effects of providing hospital healthcare during SARS outbreak. Emerg. Infect. Dis. 12, 1924-1932. doi: 10.3201/eid1212.060584

Mazzocco, K., and Cherubini, P. (2010). The effect of outcome information on health professionals' spontaneous learning. Med. Educ. 44, 962-968. doi: 10. 1111/j.1365-2923.2010.03744.x

MEDSCAPE (2020). In Memoriam: Healthcare Workers Who Have Died of COVID-19. Available online at: https://www.medscape.com/viewarticle/927976 (Accessed September 7, 2020).

Monzani, D., Vergani, L., Pizzoli, S. F. M., Marton, G., Mazzocco, K., Bailo, L., et al. (2020). Sexism interacts with patient-physician gender concordance in influencing patient control preferences: findings from a vignette experimental design. Appl. Psychol. Health Well-Being 12, 471-492. doi: 10.1111/aphw.12193

Piccinelli, M., Bisoffi, G., Bon, M. G., Cunico, L., and Tansella, M. (1993). Validity and test-retest reliability of the Italian version of the 12-item General Health Questionnaire in general practice: a comparison between three scoring methods. Comprehens. Psychiatry 34, 198-205. doi: 10.1016/0010-440x(93) 90048-9

Preti, E., Di Mattei, V., Perego, G., Ferrari, F., Mazzetti, M., Taranto, P., et al. (2020). The psychological impact of epidemic and pandemic outbreaks on healthcare workers: rapid review of the evidence. Curr. Psychiatry Rep. 22:43. doi: $10.1007 / \mathrm{s} 11920-020-01166-\mathrm{z}$

Rosenbaum, L. (2020). Facing Covid-19 in Italy - Ethics, logistics, and therapeutics on the epidemic's front line. N. Eng. J. Med. 382, 1873-1875. doi: 10.1056/ NEJMp2005492

Sadler, W. A., and Weiss, R. S. (1975). Loneliness: the experience of emotional and social isolation. Contemporary Soc. 4, 171-173. doi: 10.2307/2062224

Shanafelt, T., Ripp, J., and Trockel, M. (2020). Understanding and addressing sources of anxiety among health care professionals during the COVID-19 pandemic. JAMA - J. Am. Med. Assoc. 323, 2133-2134. doi: 10.1001/jama.2020.5893

Shankar, A., Hamer, M., McMunn, A., and Steptoe, A. (2013). Social isolation and loneliness: relationships with cognitive function. Encyclopedia Mental Health 75, 161-170. doi: 10.1016/B978-0-12-397045-9.00118-

Simard, S., Thewes, B., Humphris, G., Dixon, M., Hayden, C., Mireskandari, S., et al. (2013). Fear of cancer recurrence in adult cancer survivors: a systematic review of quantitative studies. J. Cancer Surviv. 7, 300-322. doi: 10.1007/ s11764-013-0272-z

Sorkin, D., Rook, K. S., and Lu, J. L. (2002). Loneliness, lack of emotional support, lack of companionship, and the likelihood of having a heart condition in an elderly sample. Ann. Behav. Med 24, 290-298. doi: 10.1207/ S15324796ABM2404_05

Suñer-Soler, R., Grau-Martín, A., Flichtentrei, D., Prats, M., Braga, F., FontMayolas, S., et al. (2014). The consequences of burnout syndrome among healthcare professionals in Spain and Spanish speaking Latin American countries. Burnout Res. 1, 82-89. doi: 10.1016/j.burn.2014.07.004

Wallace, C. L., Wladkowski, S. P., Gibson, A., and White, P. (2020). Grief during the COVID-19 pandemic: considerations for palliative care providers. J. Pain Symptom Manage. 60, e70-e76. doi: 10.1016/j.jpainsymman.2020.04.012

Wang, J., Mann, F., Lloyd-Evans, B., Ma, R., and Johnson, S. (2018). Associations between loneliness and perceived social support and outcomes of mental health problems: a systematic review. BMC Psychiatry 18:156. doi: 10.1186/s12888018-1736-5

Zhu, Z., Xu, S., Wang, H., Liu, Z., Wu, J., Li, G., et al. (2020). COVID-19 in Wuhan: immediate psychological impact on 5062 health workers. MedRxiv doi: $10.1101 / 2020.02 .20 .20025338$

Conflict of Interest: The authors declare that the research was conducted in the absence of any commercial or financial relationships that could be construed as a potential conflict of interest.

Copyright (c) 2020 Marton, Vergani, Mazzocco, Garassino and Pravettoni. This is an open-access article distributed under the terms of the Creative Commons Attribution License (CC BY). The use, distribution or reproduction in other forums is permitted, provided the original author(s) and the copyright owner(s) are credited and that the original publication in this journal is cited, in accordance with accepted academic practice. No use, distribution or reproduction is permitted which does not comply with these terms. 